\title{
Study on the New Progress in the Etiology of Cervical Cancer
}

\author{
Lili Xuan ${ }^{1}$, Xiaojun Liu*, \\ ${ }^{1}$ China-Japan Union hospital of Jilin University, Changchun, Jilin, 130033 \\ *corresponding author
}

Keywords: Etiology; Cervical Cancer; new progress

\begin{abstract}
Cervical cancer is one of the most common gynecological malignancies. The incidence rate ranks second among female malignancies, only after breast cancer, but it even ranks first in some developing countries. According to statistics from around the world, there are approximately 500,000 new cases of cervical cancer each year, accounting for $5 \%$ of all new cancer cases, and $80 \%$ of these cases occur in developing countries. There are about 240,000 patients who die of cervical cancer every year in the world. In recent years, according to reports from some countries and regions, the incidence of HPV infection among young women 25 years of age or younger per year is $2.3 \%$. Of these, $0.03 \%$ of infected individuals develop CIN III and cervical infiltrating cancer. The younger age of HPV infection affects the etiology of cervical cancer. Therefore, research is particularly important.
\end{abstract}

\section{Introduction}

Cervical cancer is the most common gynecologic malignancy, second only to breast cancer. The patient's age distribution was bimodal, ranging from 35 to 39 years old and 60 to 64 years old, with an average age of 52.2 years [1]. According to the WHO report, about 470,000 new cases of cervical cancer are diagnosed every year. About 230,000 women die from the disease each year. $80 \%$ of them occur in developing countries. In China, there are 130,000 cases, accounting for approximately the world's cervical cancer. $28 \%$ of new cases. Since cervical cancer has a long stage of precancerous lesions, and cell smears are widely used in various countries in the world, large-scale surveys are being carried out continuously. Cervical cancer can be diagnosed and treated early, and its incidence and mortality are significantly reduced, becoming tumors. The model of prevention and treatment has strengthened human confidence in overcoming cancer. In recent years, due to human papillomavirus (HPV) infection, prolificity, sexual behavior, economic status, smoking, long-term use of oral contraceptives and other factors, the incidence of cervical cancer has increased significantly in some regions. Trends, and patients tend to be younger.

\section{Etiological Characteristics}

In the past 10 years, large-scale population-based epidemiological data have shown that HPV DNA is present in more than 99\% of cervical cancers. HPV infection is the primary cause of cervical cancer and is an initiating factor [2]. There are more than 100 types of HPV subtypes, and about 20 of them are related to human skin mucosal lesions. According to its risk of tumorigenicity to the reproductive system, it is divided into "high-risk group" and "low-risk group". In the low-risk group, there were HPV-6, 11, 40, 42 and so on, which mainly caused benign hyperplasia such as condyloma acuminatum. In the high-risk group, HPV-16, 18, 31, 33, 51, etc., often caused a malignant transformation. HPV-16 mainly causes squamous cell carcinoma and HPV-18 mainly causes adenocarcinoma [3]. In Liang et al. [4], the HPV positive rate was $45.9 \%$, which is about the same as the overall infection rate in China. The integration of HPV DNA into the host cell genome plays a key role in the process of HPV-induced cervical cancer [5]. In the past 30 years, the incidence of HPV infection in the reproductive tract has increased significantly. The incidence of cervical cancer has also increased in some regions. It can be seen that HPV has played a crucial role in the pathogenesis of cervical cancer in Chinese women. 
HSV-2 was the first virus to be considered to play an important role in the process of cervical cancer induction. As early as 1968, Raw ls et al. proposed that HSV-2 may be the cause of cervical cancer. The concept was even more emphasized after the anti-HSV-2 antibody in the blood of women with cervical cancer was significantly increased. Studies have shown that patients with cervical cancer have significantly higher levels of HSV-2 antibodies than controls. HSV-2 antibody tests are $80 \%$ to $100 \%$ positive in patients with invasive cervical cancer, $50 \%$ positive for in situ cancer, and $25 \%$ positive for atypical hyperplasia. Only $20 \%$ were positive in the control group. According to Zheng Yumin's study of 195 cases of cervical cancer and 75 normal women, the detection rate of HSV-2 was $30.7 \%$, while the detection rate of the control group was $6.6 \%$. At present, some scholars believe that HSV-2 may have a synergistic carcinogenic effect with high-risk human papillomavirus. HSV-2 acts as a "promoter" repeatedly on cervical epithelial cells and HPV-16 and 18 as "promoters" cause cancerous cells to become cancerous.

Women infected with human immunodeficiency virus (HIV) are more likely to develop cervical intraepithelial neoplasia (CIN) and invasive cervical cancer than the general population. Ellerbrock et al. (1999) showed that HIV-infected women have a $20 \%$ risk of developing CIN. In contrast, the risk of developing non-HI V-infected women as CI is $5 \%$. Friedek et al. demonstrated that Chlamydia trachomatis and the genital ureaplasma urealyticum infection are associated with low-grade intraepithelial neoplasia in high-risk HPV-positive women. Wiikkl et al. performed HPV, actinomycete, herpes simplex virus type 2, vaginal trichomoniasis, and yeast examinations on 19,114 women. The standardized incidence of vaginal trichomoniasis, HPV, and herpes simplex virus type 2 was 6.4 . \%, 5.5\%, $12.0 \%$, that vaginal trichomoniasis, HPV and herpes simplex virus type 2 infection are associated with cervical canceration. Therefore, scholars at home and abroad believe that the risk of developing cervical cancer increases as the number of microbial species of the reproductive tract infection increases. These pathogens may play a synergistic role in the pathogenesis of cervical cancer. The above-mentioned infections of various microorganisms cause various chronic inflammatory diseases of the cervix, such as: severe cervical erosion, chronic cervicitis, cervical polyps, cervical condyloma, etc., which are related to the occurrence of cervical cancer, and have the potential risk of cancer. Cervical erosion is a precancerous lesion. It is the chronic inflammation with the highest incidence of cervical lesions in recent years. The relationship with cervical cancer has been confirmed several times. The pathogenesis is cervical erosion-atypical hyperplasia-carcinoma in situ-infiltration. cancer.

\section{Oncogene and Cervical Cancer}

Modern studies of tumor molecular biology have shown that various carcinogenic factors can activate cellular proto-oncogenes through different mechanisms, resulting in altered or abnormal gene structure, production of specific transforming proteins, and inducing imbalances in cell proliferation and differentiation. Metabolic abnormalities eventually lead to cell carcinogenesis. The malignant transformation of cells is a step-by-step process that is a complex biological process. Therefore, cancer can be said to be a genetic disease. Huang Guangqi et al. applied the exogenous DNA transfection technology to successfully isolate the cloned cervical cancer oncogene and established a human cervical cancer transformed cell line. Through molecular hybridization, it has been demonstrated that the oncogenes of the cervix cancer have a homologous sequence with Ha-ras, indicating that the Ha-ras proto-oncogene has been activated in cervical cancer. For the first time, PCR technology was applied to study the activation mechanism of cervical cancer oncogene. Ha-ras point mutations are one of the main modes of activation, and point mutations are observed in relation to clinical stage. The mutation rate of stage III cervical cancer was significantly higher than that of stage I and stage II, indicating that the midpoint mutation in cervical cancer may be an advanced event, which is related to the degree of malignancy and invasion and metastasis of the tumor. The study of C-erbB2 gene amplification and rearrangement in cervical cancer found that the amplification rate was $73.3 \%$, the amplification multiple was up to 16 times, and was related to the stage and degree of differentiation, and a multiple of stage III amplification was observed. Significantly higher than stage I, the poorly differentiated squamous cell carcinoma has a higher 
fold expansion than well-differentiated and C-erbB2 is found to have a gene rearrangement in cervical cancer. Because of its high frequency of amplification and rearrangement, it is suggested that C-erbB2 may play an important role in the occurrence and development of cervical cancer.

\section{Tumor Cell Apoptosis and Cervical Cancer}

Apoptosis is the natural physiological death of cells. It is an important mechanism for maintaining the normal morphous structure and function of individual tissues and organs. It is characterized by chromatin condensation, cytoplasmic fragmentation, formation of apoptotic bodies, and DNA fragmentation. Apoptosis is often referred to as programmed cell death, but the former is a morphological concept and the latter is a functional concept. If programmed cell death occurs, cells die, and cells build up and tumors develop. Recently, a large number of in vitro studies have confirmed that tumor cell apoptosis is regulated by the expression of oncogenes and tumor suppressor genes. The oncogenes and tumor suppressor genes involved in the regulation of tumor cells include C-myc, bcl-2, and P53.

Many studies have shown that the dysregulation or overexpression of C-myc gene plays a key role in all stages of tumorigenesis. The C-myc gene expression product has two regulatory cell functions: proliferation and apoptosis. Under normal circumstances, the proper expression of C-myc can make the cells in a normal state of proliferation and apoptosis and maintain the intracellular environmental balance, such as being stimulated by certain stimulating factors or signals (such as certain growth factors, oncogenes, etc.). Balance will be destroyed. The C-myc gene is a gene involved in cell proliferation. It has been amplified in cervical inflammatory hyperplasia. In the course of canceration, cells are immortalized through gene amplification and become immortalized, leading to canceration. Recent studies have found that bcl-2 is a key regulator of physiological or pathological apoptosis. Bcl-2 can prevent apoptosis and prolong cell survival. Overexpression of bcl-2 protein affects cell proliferation while minimizing cell death, indicating that bcl-2 is a novel oncogene. Cell proliferation and cell death and cell accumulation can be expressed by the following formula: Cell accumulation rate $=$ Cell proliferation rate - Cell mortality This formula indicates that tumorigenesis is associated with increased cell proliferation or decreased cell death. Therefore, genes that reduce cell death, such as bcl-2, and oncogenes that increase cell proliferation are at least equally important in the development of cancer. P53 is one of the most widely studied tumor suppressor genes. The study of P53 gene and its product, P53 protein, is a hot topic in the field of tumor research. It is confirmed that $50 \%$ of human malignant tumors are related to P53, and mutation of P53 gene is a tumor cell. The most common gene mutation in China, this mutation leads to disordered cell growth and reproduction. P53 gene acts as a tumor suppressor gene involved in the growth and downregulation of normal and tumor cells, and its mechanism of action is through affecting the cellular response to DNA damage, resulting in cell arrest in G1 phase and promoting apoptosis of abnormal cells. In the study of the relationship between P53 and cervical cancer, the researchers first discovered that HPVE6 protein can combine with P53 protein to form a complex. The process requires the participation of an additional cytokine called E6-related protein, E6 and After the Wtp53 protein is coupled, the Wtp53 protein will be degraded through a ubiquitous proteolytic pathway. A decrease in Wtp53 levels was observed in HPV16, HPV18, and HPV31 positive cell lines, with a half-life reduction of 50\% and 70\%. Some analyses of P53 in 92 cases of cervical carcinoma in situ revealed that allele imbalance was observed in $22 \%$ of patients, and overexpression of $\mathrm{P} 53$ was observed in $55 \%$ of cases.

\section{Conclusion}

In summary, the pathogenic factors of cervical cancer are complex. Cervical erosion, sexual confusion, poor sanitation, and high-risk sexual partners can cause cancer-causing factors (such as HSV-2, HPV, HCMV, etc.) to pass into the cervix through sexual contact, thereby increasing inflammation or erosion of the cervix. Erosion is the basis of carcinogenesis. In the process of erosion repair, the frequency of proliferation of cervical reserve cells can be increased, followed by 
delayed differentiation or differentiation, leading to canceration. Studies of tumor molecular biology in modern times have shown that the occurrence and development of cancer involve a variety of gene structure changes or abnormal expressions. In cervical cancer, Ha-ras, C-erbB2, C-myc and other genes have already existed, which are amplified and amplified by point mutations. Different mechanisms, such as platooning, are activated and play a synergistic carcinogenic role in cervical canceration. In the process of carcinogenesis and oncogene dominant carcinogenesis, equally important is the structural changes or loss of function of tumor suppressor genes. In recent years, researchers have proposed the concept of programmed cell death or apoptosis. If apoptosis occurs, cells will accumulate and cause tumors. The apoptosis of cancer cells is regulated by the expression of oncogenes and tumor suppressor genes. At present, the more positive apoptotic regulatory factors are C-myc, bcl-2, and P53. The proper expression of C-myc oncogene and P53 tumor suppressor gene can initiate or stimulate apoptosis, but some factors such as bcl-2, HPV cause C-myc overexpression or P53 mutation to prevent apoptosis and cause cell proliferation. The tumor has a certain causal relationship. The in-depth study of the etiology of cervical cancer will increase our understanding of the causes of cervical cancer, actively treat cervical precancerous lesions, and reduce the incidence of cervical cancer.

\section{References}

[1] Li Tingfang, Chen Rui. Epidemiology of Cervical Cancer[J]. Practical Journal of Clinical Medicine, 2005 .2(2):19.

[2] S ILVA D M, EIBEN C 'L, FAUSCH S C, et al. Cervical cancervaccines: emerging concepts and developments [J]. Cell Physiol, 2001, 186(2): 169-182.

[3] Ma Ding, Ruan Ling. The epidemiology and etiology of cervical cancer [J]. Journal of Practical Obstetrics and Gynecology, 2001, 17 (2): 61-62.

[4] Liang Lizhi, Yan Liangqing, Huang Xin, et al. Clinical analysis of 235 cervical cancer patients under 35 years of age [J]. Chinese Journal of Gynecology and Obstetrics, 2000, 16 (9): 545.

[5] ANDE RSSON S, SAFARI H, M INTS M, et al. Type distribu tion, viral load and integration status of high-risk human papillomaviruses in pre-stages of cervical cancer (CIN) [J]. Br J Cancer, 2005, 92(12):2195-2200. 